The Astrophysical Journal, 526:631-642, 1999 December 1

(c) 1999. The American Astronomical Society. All rights reserved. Printed in U.S.A.

\title{
MAGNETOCENTRIFUGAL LAUNCHING OF JETS FROM ACCRETION DISKS. I. COLD AXISYMMETRIC FLOWS
}

\author{
RUBEN KRASNOPOLSKY \\ Theoretical Astrophysics, California Institute of Technology, 130-33 Caltech, Pasadena, CA 91125; ruben@tapir.caltech.edu \\ ZHI-YUN LI \\ Astronomy Department, University of Virginia, Charlottesville, VA 22903; z14h@protostar.astro.virginia.edu \\ AND \\ ROGER BLANDFORD \\ Theoretical Astrophysics, California Institute of Technology, 130-33 Caltech, Pasadena, CA 91125; rdb@tapir.caltech.edu \\ Received 1999 February 15 ; accepted 1999 July 19
}

\begin{abstract}
We present time-dependent, numerical simulations of the magnetocentrifugal model for jet formation, in an axisymmetric geometry, using a modification of the ZEUS3D code adapted to parallel computers. The gas is supposed cold with negligible thermal pressure throughout. The number of boundary conditions imposed on the disk surface is that necessary and sufficient to take into account information propagating upstream from the fast and Alfvén critical surfaces, avoiding overdetermination of the flow and unphysical effects, such as numerical "boundary layers" that otherwise isolate the disk from the flow and produce impulsive accelerations.

It is known that open magnetic field lines can either trap or propel the gas, depending upon the inclination angle, $\theta$, of the poloidal field to the disk normal. This inclination is free to adjust, changing from trapping to propelling when $\theta$ is larger than $\theta_{c} \sim 30^{\circ}$; however, the ejected mass flux is imposed in these simulations as a function of the radius alone. As there is a region, near the origin, where the inclination of field lines to the axis is too small to drive a centrifugal wind, we inject a thin, axial jet, expected to form electromagnetically near black holes in active galactic nuclei and Galactic superluminal sources.

Rapid acceleration and collimation of the flow is generally observed when the disk field configuration is propelling. We parameterize our runs using a magnetic flux $\Psi \propto R^{-e \Psi}$ and mass flux $j=\rho v_{z} \propto R^{-e_{j}}$. We show in detail the steady state of a reference run with parameters $e_{\Psi}=-1 / 2, e_{j}=3 / 2$, finding that the wind leaves the computational volume in the axial direction with an Alfvén number $M_{\mathrm{A}} \sim 4$, poloidal speed $v_{p} \sim 1.6 v_{K 0}$, collimated inside an angle $\theta \sim 11^{\circ}$. We show also the thrust $T$, energy $L$, torque $G$, and mass discharge $\dot{M}$ of the outgoing wind, and we illustrate the dependence of these quantities with the exponents $e_{\Psi}$ and $e_{j}$.

Subject headings: galaxies: active - ISM: jets and outflows - methods: numerical - MHD
\end{abstract}

\section{INTRODUCTION}

Astrophysical jets are fast and well-collimated flows, observed in a wide variety of astronomical systems, ranging from young stellar objects (e.g., Lada 1985), Galactic superluminal sources (e.g., Mirabel \& Rodríguez 1998; Hjellming 1997), to quasars (e.g., Begelman, Blandford, \& Rees 1984). A single mechanism may be responsible for their formation and collimation (e.g., Livio 1997). A candidate model is the magnetocentrifugal mechanism proposed by Blandford \& Payne (1982), investigated for instance in Clarke, Norman, \& Burns (1986), Lovelace et al. (1986), Pudritz \& Norman (1986), Königl (1989), Ostriker (1997), and reviewed recently by Königl \& Pudritz (2000; see, however, Fiege \& Henriksen 1996 for an alternative model). In this picture, parcels of cold gas are stripped from the surface of a Keplerian disk and flung out along open magnetic field lines by centrifugal force. At large distances from the source region, rotation winds the field lines up into concentric loops around the axis. These magnetic loops pinch on the outflow (or wind) and collimate it into a narrow jet.

The original wind solution of Blandford \& Payne (1982) is axisymmetric, steady state, and self-similar. The selfsimilarity Ansatz is expected to break down near the rotation axis and at large distances, where appropriate boundary conditions must be imposed. There have been several studies seeking non-self-similar steady state wind solutions. Sakurai $(1985,1987)$ and Najita \& Shu (1994) took the approach of starting directly from the timeindependent MHD equations and found steady state solutions iteratively. However, no general solutions are available at the present from this approach, due to the great complexity of the critical surfaces whose loci are unknown a priori (Heinemann \& Olbert 1978). Moreover, there is no guarantee that a steady state solution can be found at all for a given set of boundary conditions. A time-dependent approach provides a surer method for studying MHD outflows, both steady state or not. This more flexible approach has been adopted by several authors (e.g., Lind et al. 1989; Stone \& Norman 1993a, 1993b; Ouyed \& Pudritz 1997; Romanova et al. 1997; Ustyugova et al. 1999; Keppens \& Goedbloed 1999; Bogovalov \& Tsinganos 1999). It is also the approach we shall take.

Following Stone \& Norman (1993a, 1993b) and Ouyed \& Pudritz (1997), we treat the Keplerian disk as a boundary and use the Zeus MHD code to simulate the timedependent disk wind. The main subtleties come from treating the disk-wind boundary - the disk surface - and the region close to the rotation axis. A special treatment of the 
disk-wind boundary is warranted, because this is the place where matter is ejected into the wind and where magnetic field lines are anchored. On this boundary, some of the flow variables can be fixed, but not all of them. This point is illustrated most clearly by steady state MHD winds, which have three well-known critical surfaces-slow, Alfvénic, and fast (Heinemann \& Olbert 1978). Crossing each of these critical surfaces imposes a set of constraints on the flow variables. Similarly, it is impossible to assign arbitrary fixed values to all the quantities at the disk boundary: some quantities must be left free to adjust according to flow conditions in the subfast region. Failure to do so will overdetermine the problem, forming numerical boundary layers with local discontinuities and impulsive acceleration of numerical origin close to the disk, which may cast doubts about the origin of the jet launched in the simulation. A numerical boundary layer may cause the effective boundary conditions governing the wind flow to differ from those imposed at the disk, as mentioned in Meier et al. (1997). This loss of control is particularly inconvenient when doing parametrical studies. In this paper we prevent such overdeterminations by properly taking into account the existence of information going upstream from the critical surfaces. Our main improvement over previous Zeus-based works is to fix only the necessary number of boundary conditions at the disk surface. Romanova et al. (1997) and Ustyugova et al. (1999) have implemented a similar method for their Godunov-type code independently.

In addition, we derive a physically motivated method for treating the outflow near the rotation axis. Centrifugal acceleration in the axial region does not happen in the magnetocentrifugal picture because the centrifugal force vanishes on the axis. A gravitational infall is expected along the axis, which could disrupt the outflow from the disk through streaming instabilities. To prevent such infall, some previous authors (e.g., Ouyed \& Pudritz 1997) assumed a high (thermal and/or turbulent) pressure to counterbalance the gravity of the central object. We, on the other hand, assume the presence of a narrow jet near the axis as explained in $\S 2.2$. Such a jet could be driven either electromagnetically by a rotating black hole (Blandford \& Znajek 1977) in the context of active galactic nuclei (AGNs) or by interaction of a central star with a disk stellar wind (e.g., Shu et al. 1991) in the context of young stellar objects.

In this paper we describe in detail our numerical approach and present our first results using the code. In $\S 2$ we explain the physical model, the numerical code is introduced in $\S 3.1$ and the boundary conditions in $\S 3.2$. Our results are described in $\S 4$, and conclusions in $\S 5$. Further features of the numerical approach are presented in an Appendix.

In forthcoming papers we shall explore the effects of the size of the jet-launching disk and the dependence of the mass flux $j$ with the inclination angle $\theta$, and perform a three-dimensional study.

\section{PHYSICAL MODEL}

\subsection{Equations and Notation}

We will use axisymmetric cylindrical coordinates $(z, R$, $\phi)$, such that the launching surface is at $z=0$ and the axis at $R=0$. A subindex $p$ will denote quantities in the poloidal plane $(z, R)$. The notation is similar to that in Blandford \& Payne (1982) or Lovelace et al. (1986).
The adiabatic MHD equations used here are

$$
\begin{aligned}
\frac{\partial \rho}{\partial t}+\nabla \cdot(\rho v) & =0 \\
\rho \frac{\partial v}{\partial t}+\rho(v \cdot \nabla) v & =-\nabla p+\rho \nabla \Phi_{g}+j \times \frac{B}{c}, \\
\frac{\partial \boldsymbol{B}}{\partial t} & =\nabla \times(v \times B)=\nabla \times \mathscr{E}, \\
\frac{\partial u}{\partial t}+\nabla \cdot(u v) & =-p \nabla \cdot v, \\
p & =(\gamma-1) u,
\end{aligned}
$$

where

$$
\begin{aligned}
\rho & =\text { matter density }, \\
\boldsymbol{v} & =\text { velocity flow field }, \\
\boldsymbol{B} & =\text { magnetic field }, \\
\boldsymbol{j} & =(c / 4 \pi) \boldsymbol{\nabla} \times \boldsymbol{B}=\text { current density }, \\
\mathscr{E} & \equiv \boldsymbol{v} \times \boldsymbol{B}=-c \boldsymbol{E}, \\
\Phi_{g} & =\text { gravitational potential }, \\
p & =\text { thermal pressure }, \\
u & =\text { internal energy density (per unit volume) }, \\
\gamma & =\text { adiabatic index } .
\end{aligned}
$$

We use a smoothed gravity, $\Phi_{g}=-\Omega_{0}^{2} r_{g}^{3} /\left(r_{g}^{2}+z^{2}+R^{2}\right)^{1 / 2}$, with $\Omega_{0}$ and $r_{g}$ fixed parameters. The product $v_{K 0}=\Omega_{0} r_{g}$ gives the scale of Keplerian speeds.

Some quantities are conserved along the field lines in the steady state (Mestel 1968), becoming functions of the magnetic flux $\Psi$ alone. Two such quantities are the relation between mass and magnetic flux $k=4 \pi \rho v_{p} / B_{p}$ and the conserved angular velocity $\Omega=R^{-1}\left(v_{\phi}-B_{\phi} v_{p} / B_{p}\right)$, which at $z=0$ has the equilibrium value $\Omega=\left(R^{\underline{1}} \partial \Phi_{g} / \partial R\right)^{1 / 2} \equiv$ $v_{K} / R$. In steady state, the velocity is related to the magnetic field by $\boldsymbol{v}=(k \boldsymbol{B} / 4 \pi \rho)+R \Omega \hat{\phi}$. Conservation also applies to the specific angular momentum $\ell=R\left(v_{\phi}-B_{\phi} / k\right)$ and the specific energy $e=v^{2} / 2+h+\Phi_{g}-\Omega R B_{\phi} / k$, where $h$ is the enthalpy, of small importance in our cold winds. Finally, the entropy $S$ is conserved by our equations, but it is not relevant here. We will check these conserved quantities as a measure of the final approach to steady state.

\subsection{Magnetocentrifugal Acceleration Model}

Consider poloidal magnetic field lines emerging from the surface of an accretion disk. In steady state, the flow will be along these lines, due to the frozen-in property of ideal MHD flows. We can picture one element of gas flowing along a field line as a "bead" sliding on a rigid "wire" (Henriksen \& Rayburn 1971). Let that bead start from $z=0$, at a slow speed or at rest. The wire is rotating at a constant angular speed $\Omega$. This will exert on the bead a centrifugal force outward, competing against the gravitational force pushing the bead inward. For a lever arm large enough, the centrifugal force wins this competition, and the field line propels the flow. This happens when the inclina- 
tion $\theta$ of the poloidal field lines from the axis is larger that a critical angle $\theta_{c}$, allowing the flow to accelerate centrifugally along the field line as if shot by a sling. This critical angle is $\theta_{c}=30^{\circ}$ for Newtonian gravity and Keplerian rotation, only slightly changed by our smoothed gravity.

The field lines twist in the toroidal direction due to the differential rotation between the corona and the disk. This generates a toroidal component $B_{\phi}$, whose magnetic pressure tends to collimate the flow, decreasing $\theta$ along a field line for increasing height $z$. In addition, the gradient in the poloidal field pressure causes focusing, depending on the shape of the flux function. Critical surfaces are the Alfvénic $\left[v_{p}=B_{p} /(4 \pi \rho)^{1 / 2} \equiv v_{A p}\right]$ and fast surface $\left[v_{p}=B /(4 \pi \rho)^{1 / 2} \equiv\right.$ $\left.v_{A t}\right]$. The slow magnetosonic surface is not relevant here due to our low sound speed. Acceleration is due to the centrifugal and magnetic forces, which, taken per unit mass and projected along the poloidal field lines, are $f_{C}=\left(v_{\phi}^{2} / R\right) \sin \theta$ and $f_{M}=(\boldsymbol{j} \times \boldsymbol{B}) \boldsymbol{B}_{p} / c \rho B_{p}=-\left(1 / 8 \pi \rho R^{2}\right)\left(\boldsymbol{B}_{p} / B_{p}\right) \nabla\left(R B_{\phi}\right)^{2}$ (Ustyugova et al. 1999). These two forces must counter the projected gravitational pull $f_{g}=-\left(\boldsymbol{B}_{p} / B_{p}\right) \nabla \Phi_{g}$. Effective acceleration requires that the collimation of the field lines is not complete before the Alfvén surface is reached: otherwise the projected centrifugal force would be too small.

Close to the axis, the field lines have an inclination lower than the critical angle $\theta_{c}=30^{\circ}$, so they cannot propel the flow centrifugally. For the purpose of designing this fully cold simulation, we cannot prevent gravitational infall by the use of thermal pressure, which is an isotropic mechanism that would push across field lines as well as along them, making a large fraction of our simulation hot. We assume instead a ballistic axial injection of matter close to escape speed. Its ram pressure provides a simple nonisotropic mechanism, which achieves the numerical objective of stopping gravitational backflow and isolating the region where the inclination of field lines is too small, without compromising the footpoints of the field lines with transverse flows, as a pressure-driven mechanism might tend to do. This jet core made of axial flow is not only a convenient numerical device; it is physically justified in AGNs, and galactic superluminal sources, which may create a central electromagnetic jet carrying Poynting flux and exerting an anisotropic Maxwell stress at its surface. (Blandford \& Znajek 1977). The ballistic injection thus represents the effects of a feature of the flow not covered by the magnetocentrifugal model alone.

\section{NUMERICAL METHODS}

\subsection{Computer Code}

To perform our simulations we wrote a variant, called ZEus36, based on the ZEUS3D code, released by LCA (Clarke, Norman, \& Fiedler 1994; Norman 1996; Stone \& Norman 1992a, 1992b). Zeus3D is an explicit, finitedifferencing algorithm, running on an Eulerian grid. Conservation of mass and momentum components is guaranteed by the code. Magnetic fields are evolved using the constrained transport method (Evans \& Hawley 1988), which guarantees that no monopoles will be generated, limited only by the numerical precision of the machine. An advanced method of characteristics (Hawley \& Stone 1995) is used to calculate $\mathscr{E}=\boldsymbol{v} \times \boldsymbol{B}$ and the transverse Lorentz force. A large base of tests has already been done on this community code, and we have not changed the central difference scheme.
ZEUs36 is a modification of ZeUs3D written for parallel computers, such as for instance the Intel Paragon and the Touchstone Delta. It uses MPI-a standard message passing interface for parallel machines, including networks of workstations. This makes Zeus36 easily portable in the parallel computing world. The three-dimensional parallelization scheme is straightforward. We subdivide the computational cubic grid into $N$ smaller grids, each given to one of the $N$ compute nodes. Communication between the nodes is realized by taking advantage of the boundary condition subroutines, appropriately modified to allow communication between adjacent compute nodes. Zeus36 also improves upon the latest released versions of Zeus3D (version 3.4) by treating the boundary conditions at the corners and edges of the grid more consistently. Corners and edges are of less importance when the code is not run in parallel. They become more important in a parallel treatment, where they appear in the middle of the overall mesh rather than less obtrusively at the boundaries of the calculation. The user interface was modified to make it more convenient to our usage on many different computer systems, serial and parallel. For instance, it was decided not to use the powerful precompiler EDITOR (Clarke 1992), bundled into the Zeus3D release. These changes, together with the use of dynamic memory allocation, eliminate the need to recompile the main code when the grid size is changed.

ZEUS36 has been tested for both internal consistency and consistency with ZEUS3D. We have made sure that the output does not depend on the distribution or number of the nodes used to compute a given grid. To test consistency with ZEUs3D, we have run some test problems using both programs, and compared their output byte-by-byte. The small departures observed were those due to the improvement in the treatment of corners and edges: no difference appeared after those were accounted for.

\subsection{Boundary Conditions}

In these axisymmetric simulations the computational grid has four boundaries: axis $(R=0)$, outer radius $(R=$ $\left.R_{\max }\right)$, disk $(z=0)$ and outer height $\left(z=z_{\max }\right)$. The code enforces boundary conditions by assigning values to the fields $\rho, u, v$, and $\mathscr{E}$ at the grid edge and at a few ghost zones outside the computational grid. The boundary conditions for $\boldsymbol{B}$ are enforced indirectly from the values of $\mathscr{E}$, using the equation $\partial \boldsymbol{B} / \partial t=\boldsymbol{\nabla} \times \mathscr{E}$ to evolve $\boldsymbol{B}$ in both active and ghost zones, ensuring $\nabla \cdot \boldsymbol{B}=0$ everywhere. The exact location and grid staggering of these ghost zones follows Stone \& Norman (1992a, 1992b).

$$
\text { 3.2.1. } R=0
$$

The axis boundary is geometrically well determined by axisymmetry, and it has offered no complications either in implementing or running. The conditions are implemented as usual for the axis in cylindrical coordinates. In the calculation of ghost zone values for $R<0$, scalar fields are reflected, and vector components are reflected with a change of sign in the directions $R$ and $\phi$.

$$
\text { 3.2.2. } z=z_{\max }
$$

The upper boundary is treated in our simulation using an outflow boundary condition, as defined in the Zeus3D code. Specifically, for $z>z_{\max } A(z)=A\left(z_{\max }\right)$ for all fields, except for $v_{z}(z)=\max \left[v_{z}\left(z_{\max }\right), 0\right]$. This is not perfect, but, as the physics of our simulation implies the existence of a super- 
sonic, super-Alfvénic accelerated outflow, these imperfections are not expected to have a large influence in the simulated flow.

$$
\text { 3.2.3. } R=R_{\max }
$$

This is more delicate, especially at low altitude where the flow is sub-Alfvénic, and can influence the upstream flow. In practice we use similar outflow conditions as for $z=z_{\max }$. Label the field line that becomes trans-Alfvénic at $R=$ $R_{\max }$, by $\Psi=\Psi_{1}$ (Fig. 1), and it is clear that artificial conditions imposed at $R=R_{\max }$ for $\Psi>\Psi_{1}$ may induce spurious collimation. We must therefore explore the sensitivity of our results to our treatment of this part of the flow (cf. $\S 4.3$ ).

In one example of an alternative prescription, Romanova et al. (1997) have used a "force-free" prescription, $\boldsymbol{j}_{p} \| \boldsymbol{B}_{p}$, that can be written as $\boldsymbol{B}_{p} \cdot \nabla\left(R B_{\phi}\right)=0$; a later proposal from the same authors is taking $B_{p} \cdot \nabla\left(R B_{\phi}\right)=\alpha B_{R} B_{\phi}$, with $\alpha$ a constant to be determined (Ustyugova et al. 1999), observing that artificial collimation may occur for inappropriate box sizes.

$$
\text { 3.2.4. } z=0
$$

Even more important is the treatment of $z=0$. It represents the launching surface of the model, located at the

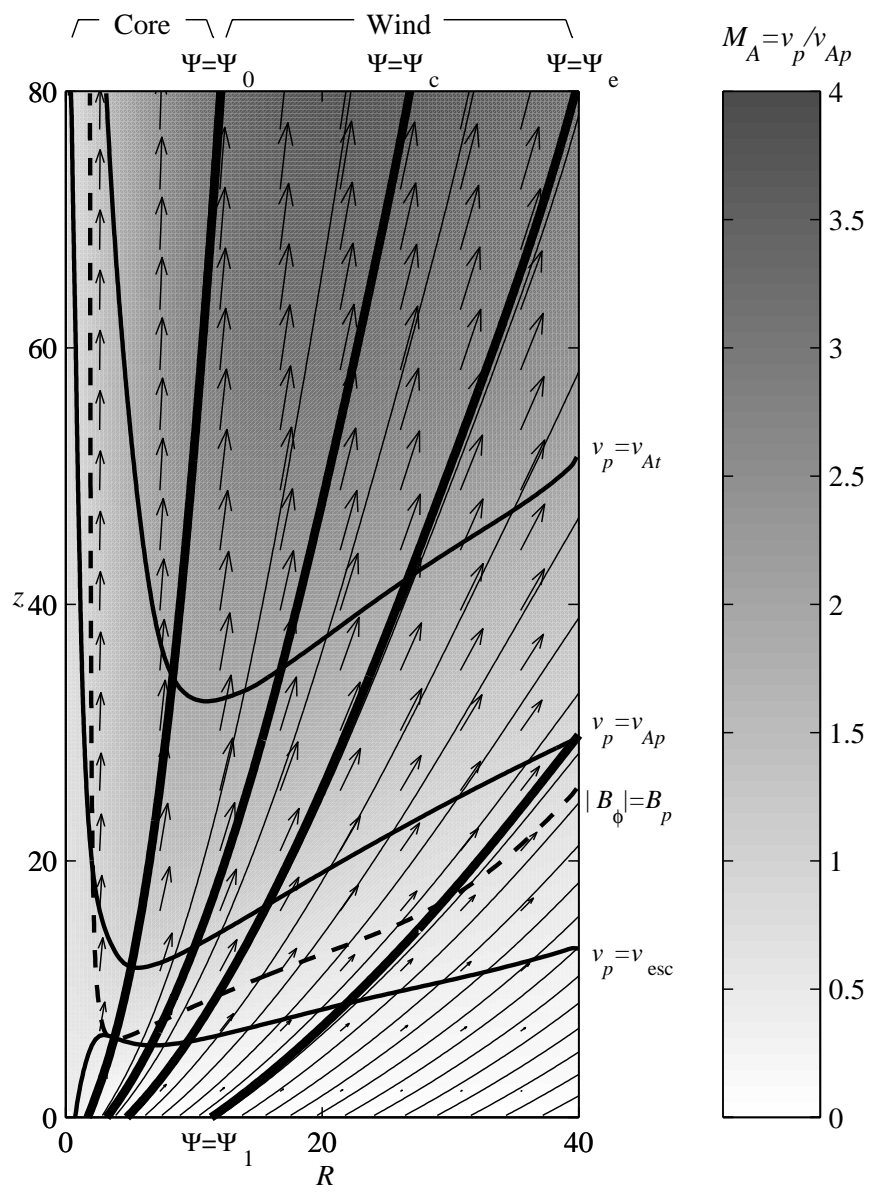

FIG. 1.-Reference simulation. The local Alfvén number $v_{p} / v_{A p}$ is shown in gray-scale. In thin black, field lines and vectors of $v_{p}$. In medium black, the critical surfaces $v_{p}=v_{A p}, v_{p}=v_{A t}$, and the local escape speed line $v_{p}=\left(-2 \Phi_{q}\right)^{1 / 2} \equiv v_{\text {esc }}$; the line $B_{p}=B_{\phi}$ is shown in dashes. In thick black, some selected field lines: $\Psi_{1}$ passing through the intersection between the Alfvén surface and the outer boundary; $\Psi_{0}$ at the outer edge of the axial injection zone, separating the injected core from the main wind; $\Psi_{c}$, the field line whose inclination is critical at $z=0$; and $\Psi_{e}$, passing through the outer edge of the grid. top of the disk, or the base of the corona, above the slow magnetosonic surface. Its properties will determine all the flow downstream.

The first question to address is the number of boundary conditions we are allowed to fix at this surface. This number is equal to the number of waves outgoing normally from the boundary, equal to the number of characteristics. We calculate it by counting the degrees of freedom of the system, subtracting all constraints. We have, in principle, seven degrees of freedom: the density, three components of $v$ and $\boldsymbol{B}$, minus the constraint $\boldsymbol{\nabla} \cdot \boldsymbol{B}=0$, and also the internal energy, because our simulation, while dynamically cold, keeps track of a small internal energy. Each crossing of a critical surface is also a constraint that will remove one degree of freedom. In principle there are three such surfaces, slow, Alfvénic, and fast; however, the initial $v_{p}$ at $z=0$ is already larger than the slow speed, so only two critical surfaces will be crossed. We are left with five independent waves out of the original seven. Five boundary conditions should be imposed. A more detailed deduction of this result can be seen for instance in Bogovalov (1997).

We choose to fix the five fields $\rho, u, \mathscr{E}_{\phi}, \mathscr{E}_{R}$, and $v_{z}$ at the disk -if launching were subslow, the presence of the slow critical surface would reduce the number of independent boundary conditions to the first four. At $z=0$, the boundary conditions for $\mathscr{E}_{\phi}$ and $\mathscr{E}_{R}$ are derived from the infinite conductivity of the disk material, giving $\mathscr{E}_{\phi}=0$ and $\mathscr{E}_{R}=$ $R \Omega B_{z}$. The condition for $\mathscr{E}_{\phi}=\left(v_{p} \times \boldsymbol{B}_{p}\right) \cdot \hat{\phi}$ implies that $v_{p} \| \boldsymbol{B}_{p}, v_{R} / B_{R}=v_{z} / B_{z}=v_{p} / B_{p}$. The condition for $\mathscr{E}_{R}=$ $v_{\phi} B_{z}-v_{z} B_{\phi}$ implies that $v_{\phi}=R \Omega+B_{\phi} v_{p} / B_{p}=$ $R \Omega+B_{\phi} v_{z} / B_{z}$. Implementation is done by assigning values to fields at the ghost zones $z \leq 0$. Density, velocity, and internal energy are defined at the ghost zones as straightforward functions of $R$, with $u=\rho c_{s 0}^{2} /(\gamma-1)$ for a small constant $c_{s 0}$. This fixes the mass flux $\rho v_{z}$; if our launching were subsonic, this quantity should be determined from the crossing of the slow critical surface.

The magnetic field evolves in time following $\partial B_{z} / \partial t=$ $R^{-1} \partial R \mathscr{E}_{\phi} / \partial R, \quad \partial B_{R} / \partial t=-\partial \mathscr{E}_{\phi} / \partial z, \quad$ and $\quad \partial B_{\phi} / \partial t=\partial \mathscr{E}_{R} /$ $\partial z-\partial \mathscr{E}_{z} / \partial R$. Fixing $\mathscr{E}_{\phi}=0$ at $z=0$ keeps $B_{z}(R)$ constant in that plane, anchoring the field lines to the disk surface. The angle $\theta=\arctan \left(B_{R} / B_{z}\right)$ between the field lines and the vertical evolves in the active zones; a similar evolution must be present in the ghost zones. Otherwise, the field lines would present sharp kinks at the base of the wind $z=0$, associated with large currents and localized forces which might mask the launching mechanism. An evolving $B_{R}$ requires an $\mathscr{E}_{\phi}(z)$ dependence in the ghost zones; knowing that $\mathscr{E}_{\phi}$ vanishes at $z=0$, we choose $\mathscr{E}_{\phi}$ to be an odd function of $z$. The resulting antisymmetric time dependence of the field $B_{z}$ and the flux $\Psi$ allows $\theta$ to vary in approximately the same way in both active and ghost zones, so that the final inclination of the field lines is decided by the flow itself and its crossing of the critical surfaces, producing a much better steady state than alternative approaches in which the field line inclination is fixed. Similarly, we make $\mathscr{E}_{R}(z)$ symmetric around its known value at $z=0$, defining $\mathscr{E}_{R}(z)=$ $2 R \Omega(R) B_{z}(R)-\mathscr{E}_{R}(-z)$ for $z<0$. (An alternative possibility here would be extrapolating the field lines inside the ghost zones; however, this is somewhat more difficult to implement.) With this choice, one term of the time dependence of $B_{\phi}$ is even in $z$. We make the other term also even, by requiring $\mathscr{E}_{z}(z)=\mathscr{E}_{z}(-z)$, which allows this quantity to vary freely. Finally, we need values for $v_{R}$ and $v_{\phi}$. We will take 
them from $v_{R}=B_{R} v_{z} / B_{z}$ and $v_{\phi}=R \Omega+B_{\phi} v_{z} / B_{z}$, which we implement using the values of $B_{z}$ and $\Omega$ at $z=0$. Following the variation of these quantities inside the ghost zones is also a possible option.

In some simulations we find occasions - early during the run, long before steady state is approached - in which $v_{z}<$ 0 at the first active zone close to the disk. In that case we modify the boundary conditions, allowing the disk to absorb the backflow, preventing the numerical artifacts shown in the Appendix. The ghost values at $z \leq 0$ for the fields $\rho, v_{R}$, and $v_{\phi}$ are taken temporarily from their values at the first active zone with $z>0, v_{z}$ is set to zero and the internal energy to $u=\rho c_{s 0}^{2} /(\gamma-1)$. Thus, backflow is absorbed and will eventually disappear.

In this paper we artificially prescribe $j=\rho v_{z}$ as a function of $R$ alone, although mass loading is effective only for $\theta>30^{\circ}$. However, in the steady state simulations presented here this inequality is satisfied for most of the disk outside the innermost region, justifying our choices after the fact. We shall return to this in a following paper, in which $j$ will be allowed to depend also on $\theta$.

The functions of radius are parameterized using a combination of exponents and softening radii:

$$
\begin{aligned}
\rho(R) & =\rho_{0} / \sqrt{\left[1+\left(R / r_{\rho}\right)^{2}\right]^{e_{\rho}}}, \\
B_{z}(R) & =B_{z 0} / \sqrt{\left[1+\left(R / r_{b}\right)^{2}\right]^{e_{b}}}, \\
\Psi(R) & = \begin{cases}2 \pi\left(B_{z 0} r_{b}^{2}\right) \frac{\left[1+\left(R / r_{b}\right)^{2}\right]^{1-e_{b} / 2}-1}{2-e_{b}}, \\
\pi\left(B_{z 0} r_{b}^{2}\right) \log \left[1+\left(R / r_{b}\right)^{2}\right]\end{cases} \\
v_{z}(R) & =\left(v_{z \text { inner }}^{v}+v_{z \text { outer }}^{v}\right)^{1 / v}, \\
v_{z \text { inner }} & =\frac{v_{z 0} / \sqrt{\left[1+\left(R / r_{v i}\right)^{2}\right]^{e_{v i}}}}{\sqrt{\left[1+\left(R / r_{v o}\right)^{2}\right]^{e_{v o}}},}, \\
v_{z \text { outer }} & =\left[f_{v o} R \Omega(R)\right] / \sqrt{\left[1+\left(R / r_{v o}\right)^{2}\right]^{e_{v o}}} .
\end{aligned}
$$

We have used $v=1$ (linear) and $v=2$ (quadratic). For large $R, v_{z} \propto R^{\left(e_{v o}+1 / 2\right)} \equiv R^{-e_{v}}, j \equiv \rho v_{z} \propto R^{-\left(e_{v}+e_{\rho}\right)} \equiv R^{-e_{j}}$, and $\Psi \propto R^{-\left(e_{b}-2\right)} \equiv R^{-e \Psi}$.

This implementation of disk boundary conditions uses some values of the fields in the corona; there is upstream propagation of information. The velocity at $z=0$ is supersonic but sub-Alfvénic; upstream propagation of waves is physically expected. A simulation that omits this effect is incomplete, and our results are, manifestly, sensitive to the treatment of the surface conditions. There is in principle a risk of numerical instability involved in using this information in the code; fortunately, our runs did not show this kind of instability.

\subsection{Initial Conditions}

Naturally, we are interested in flows that are independent of our starting conditions. It turns out that transients generally decay in a few Alfvén crossing times and do not influence the late final flow. Nevertheless, they deserve some detailed explanation.

Initially, $\boldsymbol{B}_{p}$ is defined from its boundary condition value $B_{z}(R)$ at $z=0$, and the initial position of the footpoint $\left(z=0, R=R_{0}\right)$ of a generic poloidal field line passing through a point $(z, R)$. This footpoint $R_{0}$ is found from the equation $R_{0}^{2}-\left[R-m_{\ell} r_{\ell}\left(1+z / r_{\ell}\right)^{e^{\ell}}\right] R_{0}-R m_{\ell} r_{\ell}=0$, where $B_{z 0}, r_{b}, e_{b}, r_{\ell}, e_{\ell}$, and $m_{\ell}$ are constant parameters. The length scale $r_{\ell}$ determines the location of the initial footpoint $R_{c}$ of the critical field line $\Psi_{c}$ with $\theta=\theta_{c}, e_{\ell}$ gives the initial curvature and collimation of the field lines, and $m_{t}$ sets the maximum slope $\tan \theta$ allowed for the field lines, through the prescription $\tan \theta=e_{\ell} R /\left(r_{\ell}+R / m_{\ell}\right)$ valid at $z=0$. If $m_{\ell}$ is set to infinity, all angles $\theta$ are allowed, and $R_{0}(z, R)=R /\left(1+z / r_{t}\right)^{e_{t}}$.

The initial density at $z>0$ is given by $\rho(z, R)=\eta_{\rho} \rho(z=$ $0, R)$, with $\eta_{\rho}<1$. Initial $v_{z}$ in the corona is defined similarly through $v_{z}(z, R)=\eta_{v} v_{z}(z=0, R)$. The initial value for the internal energy is $u=\rho c_{s 0}^{2} /(\gamma-1)$.

In all but one of our simulations we have set the initial value of $B_{\phi}$ to zero, and then consistently made the initial $v_{\phi}(z=0, R)=v_{K}=R \Omega(R)$. For simplicity, this initial $v_{\phi}$ is chosen as independent of $z$. Finally, the radial component of the velocity, $v_{R}$, is set to zero in the bulk of the flow, and to its boundary condition value $v_{R}=B_{R}\left(v_{z} / B_{z}\right)$ for $z \leq z_{\min }$, where $z_{\min }$ is the value of $z$ at the first zone above the disk.

\section{RESULTS}

\subsection{The Reference Simulation}

We first describe a fiducial or reference simulation which we shall use to describe the various dependencies in our results. In this simulation, we reach the steady state solution shown in Figure 1. The simulation box has $256 \times 128$ active pixels, with $0 \leq z \leq 80.0$ and $0 \leq R \leq 40.0$. Initial and boundary conditions used to produce this run are shown in Figure 2. The boundary density at $z=0$ is constant and uniform, $\rho=\rho_{0}=1$, with initial $\rho=0.1 \rho_{0}$ for $z>0$. The Keplerian velocity scale is given by $v_{K 0}=\Omega_{0} r_{g}=1$, with $r_{g}=\sqrt{3}$. The function $v_{z}(z=0, R)$ is determined by $v_{z 0}=$ $1.7, f_{v o}=0.1, r_{v i}=r_{v o}=r_{g}, e_{v i}=2, e_{v o}=1$, making $e_{v}=1.5$. Initially, $v_{z}(z>0, R)=10^{-6} v_{z}(R), B_{\phi}=0$, and $v_{\phi}=R \Omega(R)$, independent of $z$. The parameters determining $B_{p}$ are $B_{z 0}=4 v_{K 0}\left(4 \pi \rho_{0}\right)^{1 / 2}, r_{b}=r_{g}, e_{b}=3 / 2, r_{\ell}=r_{g}, e_{\ell}=1$, and $m_{\ell}=\sqrt{3}=\tan 60^{\circ}$. The initial sound speed is set to $c_{s}=0.0002$, ensuring that pressure is unimportant in this simulation. The adiabatic index is $\gamma=5 / 3$.

The flow is then evolved until a steady state is reached. Once in steady state, the flow is accelerated up to 2.77 times

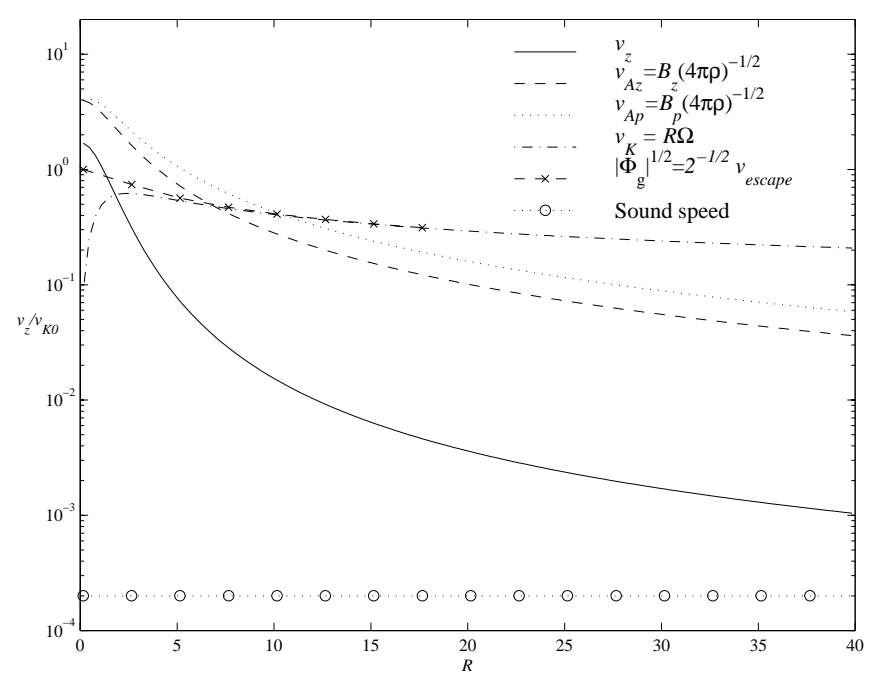

FIG. 2.- Reference simulation. Boundary and initial conditions for $v_{z}$, $v_{A z}=B_{z} /(4 \pi \rho)^{1 / 2}, v_{A p}, v_{K} \equiv R \Omega, B_{z}$, and $\left(-\Phi_{g}\right)^{1 / 2}=v_{\text {esc }} / 2^{1 / 2}$ at $z=0$ as functions of $R$, normalized by the Keplerian speed scale $v_{K 0}=\Omega_{0} r_{g}$. 
the Keplerian speed at the launching point; the acceleration is purely centrifugal and magnetic. The maximum Alfvén number $v_{p} / v_{A p}=3.98$ is found close to the upper edge, where also the maximum fast Alfvén number $v_{p} / v_{A t}$ is found, equal to 1.36. Collimation of the field lines starts even before the flow crosses the Alfvén surface $v_{p}=v_{A p}$, as we can see in Figure 1. The minimum Mach number is 140, showing that pressure is dynamically unimportant in this cold simulation. The acceleration along the field line $\Psi=$ $\Psi_{e}$ passing through the outer edge of the grid is shown in Figure 3. Important field lines are $\Psi_{1}$, the last field line able to make the Alfvén transition; $\Psi_{c}$, the field line making a critical angle $\theta_{c}$ with the axis; and $\Psi_{0}$, with footpoint at $R=r_{v i}$, at the outer edge of the "core" inner injection, thus separating this core from the wind region.

We have followed this simulation for a time equal to 2.6 Keplerian turns $2 \pi / \Omega$ at $R=R_{\max }$ (and, equivalently, 94 turns at the critical radius $R_{c}, 170$ at the smoothing length radius $r_{g}$ ). The poloidal velocity and the magnetic field change by less than $1.1 \times 10^{-4}$ in the last $10 \%$ of the run.

The approach to steady state can also be observed in the figures in the integrals of motion $\Omega, \ell, k$, and $e$, which are already functions of $\Psi$ alone, with the exception of border effects for the smallest and largest values of $z$, important mostly for the noncentrifugal core region, $\Psi<\Psi_{0}$. We followed these integrals along all field lines in the wind $\Psi>\Psi_{0}$ and found that on each field line the integrals depart from their averaged value by less than $5 \%$ for $\Omega, 6 \%$ for $\ell, 7 \%$ for $k$, and $7 \%$ for $e$ in the worst case. This still overestimates the errors; the corresponding standard deviations divided by the mean are less than $0.3 \%$ for $\Omega$, and $3 \%$ for the other integrals, further improved to $0.9 \%$ if the outermost region $\Psi>\Psi_{1}$ is excluded.

The Alfvén radius is defined by $R_{A}^{2}=\ell / \Omega$. Our parameter choice is such that angular momentum is primarily extracted from the disk by magnetic stress. Therefore, we can estimate $\ell$ for low values of $z$ by its magnetic term alone and write an approximate lever-arm ratio as $R_{A} / R_{0} \approx$ $\left[-\left(B_{\phi} / B_{z}\right) v_{A z}^{2} / v_{z} v_{K}\right]^{1 / 2}$. Figure 4 shows the observed value, the exact formula and the estimate; all agree on a value around 3 for all field lines, already large enough to justify

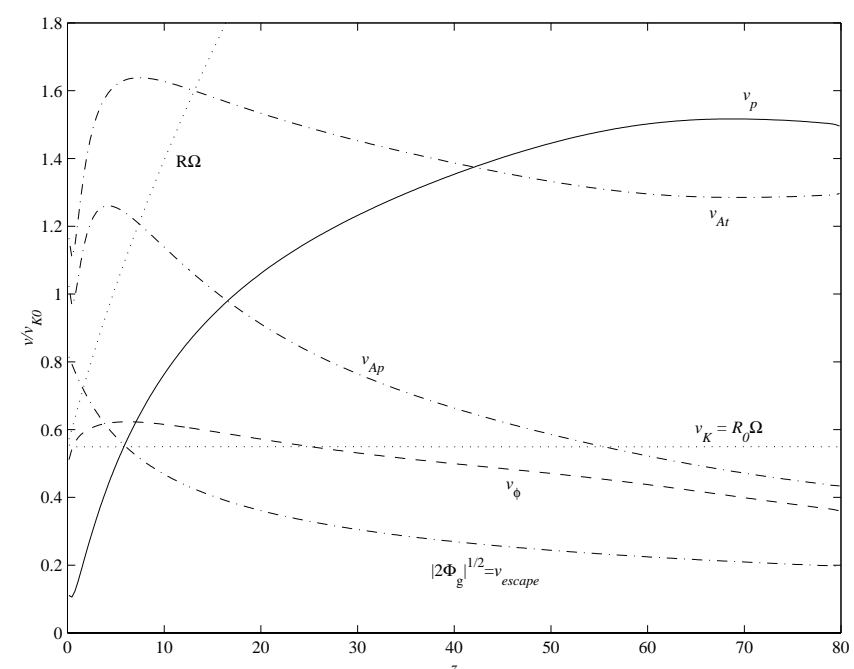

FIG. 3.-Reference simulation. Velocities along the field line $\Psi=\Psi_{e}$ passing through the outer edge of the grid. Plot of $v_{p}, v_{A p}, v_{A t}, v_{\text {esc }}, R \Omega$, $R_{0} \Omega$, and $v_{\phi}$ vs. $z$, showing the growth of poloidal speed.

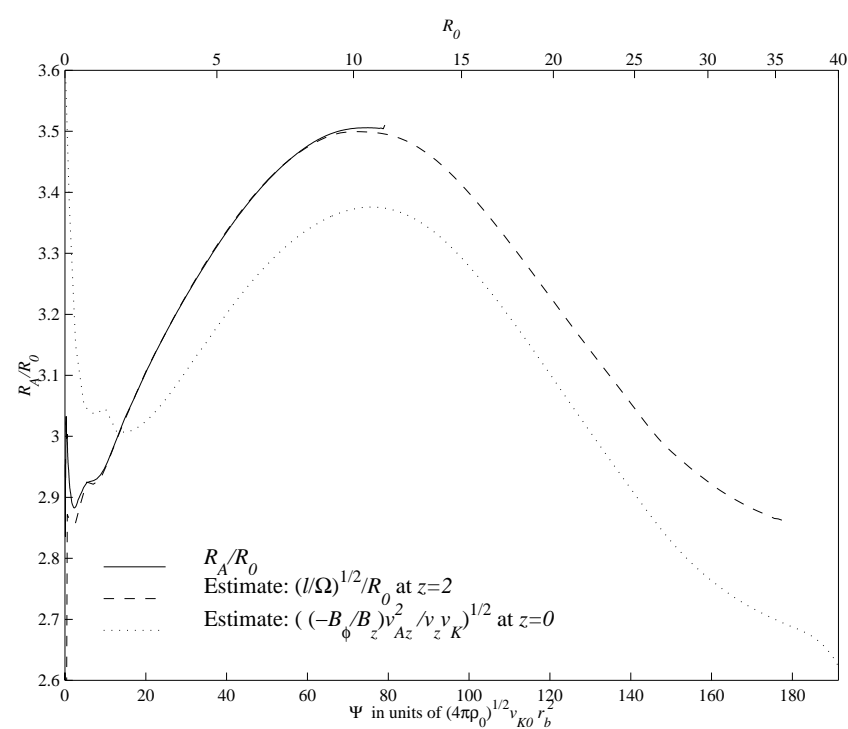

FIG. 4.-Lever arm ratio $R_{A} / R_{0}$ (solid line), compared to $(\ell / \Omega)^{1 / 2} / R_{0}$ (dashed line), and $\left[-\left(B_{\phi} / B_{z}\right) v_{A z}^{2} / v_{z} v_{K}\right]^{1 / 2}$ (dotted line). Both the flux $\Psi$ and the footpoint $R_{0}$ are used to label the field lines.

approximating $\ell$ by its magnetic term. In the formula of the estimate, the boundary conditions determine all the quantities at $z=0$, with the exception of the toroidal field $B_{\phi}$, which is allowed to self-adjust in the simulation; assuming a value for the lever-arm ratio gives an estimate for the toroidal field in steady state.

The collimation shifts the position of field line $\Psi_{c}$ making a critical angle with the disk surface. In steady state $\theta_{c}$ is found at $R_{c}=1.85 r_{\ell}$, while initially it had been $R_{c}=1.63 r_{\ell}$. The integrals $\dot{M}(\Psi), L(\Psi)$ and $G(\Psi)$ are the total fluxes of mass, energy and angular momentum between the axis and a field line $\Psi$. They have very little numerical dependence on the height $z$ of the surface used to perform the integration, because the ZeUS algorithm respects these conservation laws. The thrust $T$ is defined here as the surface integral of the kinetic energy term $\rho v_{z}^{2} / 2$, calculated at $z=z_{\max }$. The integrals $\dot{M}$ and $\Psi$ at $z=0$ are completely determined from the boundary parameters and have been done analytically. Our results are shown in Table 1.

It is important to observe that in this steady state not only the field lines and streamlines collimate, but also the density, which is roughly a function of $R$ alone for large $z$, as shown in Figure 5, in spite of the boundary condition at $z=0$, where $\rho$ is kept constant. This result is in accord with previous asymptotic analysis (Shu et al. 1995).

As a stringent quality check, we made contour plots of the ratio between the projected magnetic and centrifugal forces $f_{M}$ and $f_{C}$, defined in $\S 2.2$. Centrifugal acceleration dominates close to the disk, approximately up to the point where $v_{p}$ achieves the local escape speed; magnetic acceleration takes over afterward. There is no visible discontinuity associated with the field line $\Psi=\Psi_{1}$; this shows that the influence of the sub-Alfvénic boundary at $R=R_{\max }$ is not unduly large. However, we observe a triangle near the outer corner where $f_{M}<0$, decelerating the flow-the vertices of this triangle are the points $(z=60, R=40),(z=80$, $R=30$ ), and the outer corner. To decide if this is a physical effect or an artifact caused by the edges, we enlarged the box in both directions, as shown below in $\S 4.3 .2$, finding that it is indeed only an artifact. 
TABLE 1

Mass Discharge $\dot{M}$, Luminosity $L$, Torque $G$, and Thrust $T$ at Selected Field Lines Labeled by TheIr MAgNetic FluX $\Psi$ OR FoOtPoint $R_{0}$

\begin{tabular}{lccccccc}
\hline \hline Parameter & $\Psi_{0}$ & $\Psi_{c}$ & $\Psi_{e}$ & $2 \Psi_{c}$ & $3 \Psi_{c}$ & $\Psi_{1}$ & $R_{\max }$ \\
\hline$L \ldots \ldots \ldots \ldots$ & 5.5 & 13.1 & 18.1 & 20.5 & 24.0 & 25.0 & 28.5 \\
$G \ldots \ldots \ldots \ldots$ & 8.8 & 31 & 54 & 71 & 109 & 127 & 265 \\
$\dot{M} \ldots \ldots \ldots \ldots$ & 3.2 & 6.0 & 7.7 & 8.6 & 10.2 & 10.8 & 14.8 \\
$\Psi \ldots \ldots \ldots \ldots$ & 9.5 & 22.6 & 35.8 & 45.2 & 67.9 & 79.2 & 191 \\
$R_{0} \ldots \ldots \ldots$ & 1.73 & 3.20 & 4.77 & 6.01 & 9.41 & 11.35 & 40 \\
$R_{A} / R_{0} \ldots \ldots$ & 2.94 & 3.14 & 3.29 & 3.38 & 3.50 & 3.51 & $\ldots$ \\
$T \ldots \ldots \ldots \ldots$ & 1.9 & 4.1 & 5.5 & $\ldots$ & $\ldots$ & $\ldots$ & $\ldots$
\end{tabular}

NOTES. $-\dot{M}$ is in units of $\rho_{0} v_{K 0} r_{b}^{2}, L$ in $\rho_{0} v_{K 0}^{3} r_{b}^{2}, G$ in $\rho_{0} v_{K 0}^{2} r_{b}^{3}, T$ in $\rho_{0} v_{K 0}^{2} r_{b}^{2}$, and $\Psi$ in units of $\left(4 \pi \rho_{0}\right)^{1 / 2} v_{K 0} r_{b}^{2}$. Thrust was calculated at $z=z_{\max }$.

\subsection{Parametric Study}

We now explore the sensitivity of our solution to changes in the functions $v_{z}(R), \rho(R)$, and $B_{z}(R)$, parameterized by the exponents $e_{v}, e_{\rho}$, and $e_{b}$, or $e_{j}=e_{v}+e_{\rho}$, and $e_{\Psi}=e_{b}-2$.

First we checked that $e_{j}$ is indeed a good parameter. We did a variable density simulation with the same value of $j=\rho v_{z}$ as the standard run, but with $e_{\rho}=0.25$ instead of zero, and let it run for the same physical time. For $z>2$, the results of the variable density simulation coincide with the reference run; however, steady state is not achieved at the outer corner (Figs. 6 and 7). Despite its slower convergence, it represents the same physical steady state as the reference run, with the exception of very small values of $z$, where the different profile of $\rho$ still has some influence. For a larger

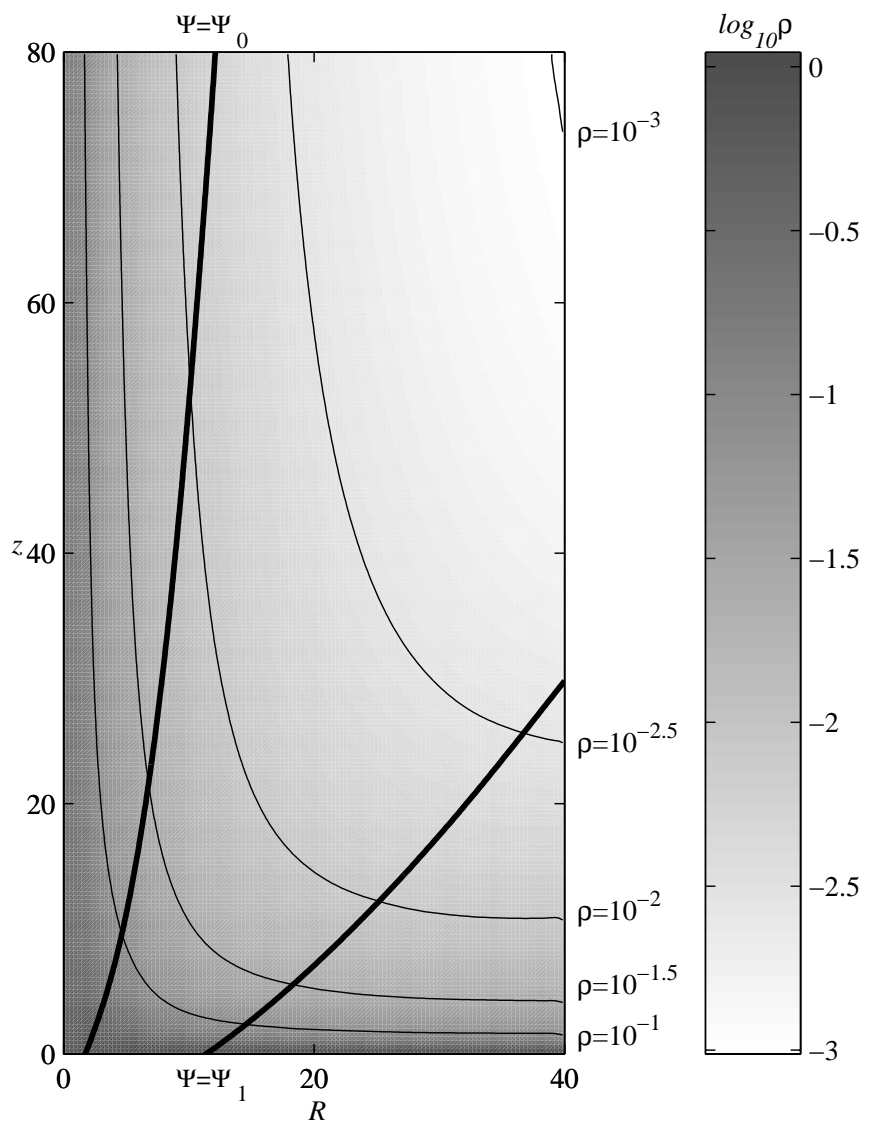

FIG. 5.-Contours and gray-scale of density in the reference run, showing collimation. value of the density exponent, such as $e_{\rho}=1$, the run fails to reach steady state in the same box as the reference run. However, simulations with a box size four times larger (see $\S$ 4.3.2) show that the $e_{\rho}=1$ case is nearly identical to the other cases. The effects of the box size are discussed in the next section. We thank the referee for suggesting this test.

Having done this, we next made a few simulations for different values of the parameters. Figure 8 shows the critical surfaces and field lines for these runs. Their shapes are qualitatively similar, with the remarkable exception of the outer field lines for the flatter $j$ simulation $e_{j}=0.5, e_{b}=1.5$; some of these lines start pointing inward instead of outward. Not all field lines are able to propel the flow centrifugally at $z=0$; they must spend some initial kinetic energy before acceleration can start. Further tests can tell if this unexpected effect is physical or only a numerical artifact. Because the assumption $|\theta|>\theta_{c}$ is not fulfilled everywhere, these tests should take into account that the mass flux should be reduced for those field lines, as we will do in future work by using a $j$ dependent on $\theta$.

Values of speed, Alfvén number, lever-arm ratio, luminosity, and torque are calculated at the point $\left(z_{\max }, R_{\max }\right)$ and given as functions of the exponents $e_{b}, e_{v}$, and $e_{\rho}$ in Table 2. The first four columns are the simulations represented in Figure 8. The last column is the variable density simulation shown in Figure 6, where we can already see that its flux at the outer corner is slightly different from that of the reference run; this is due to its insufficient convergence to the steady state in that region of the computational volume.

\subsection{Testing of Code Convergence}

We want to test the convergence of the simulations to a steady state solution by changing some computational and physical parameters from our reference run.

\subsubsection{Resolution}

We reduced the resolution of the original standard run by one half, using a box with $128 \times 64$ active pixels, $0 \leq z \leq 80.0$, and $0 \leq R \leq 40.0$. Simulation converged to a steady state similar to the reference run, with maximum $v_{p} / v_{A p}=3.74$ and maximum $v_{p} / v_{A t}=1.33$. The resolution test is therefore passed.

\subsubsection{Box Size: Changes in Both Length and Width Together}

Here we show a run done in a larger box with $256 \times 128$ active pixels, $0 \leq z \leq 160.0$, and $0 \leq R \leq 80.0$; this is the same space resolution used in $\S 4.3 .1$, with a box extending in space to twice the height and width. Once a steady state 


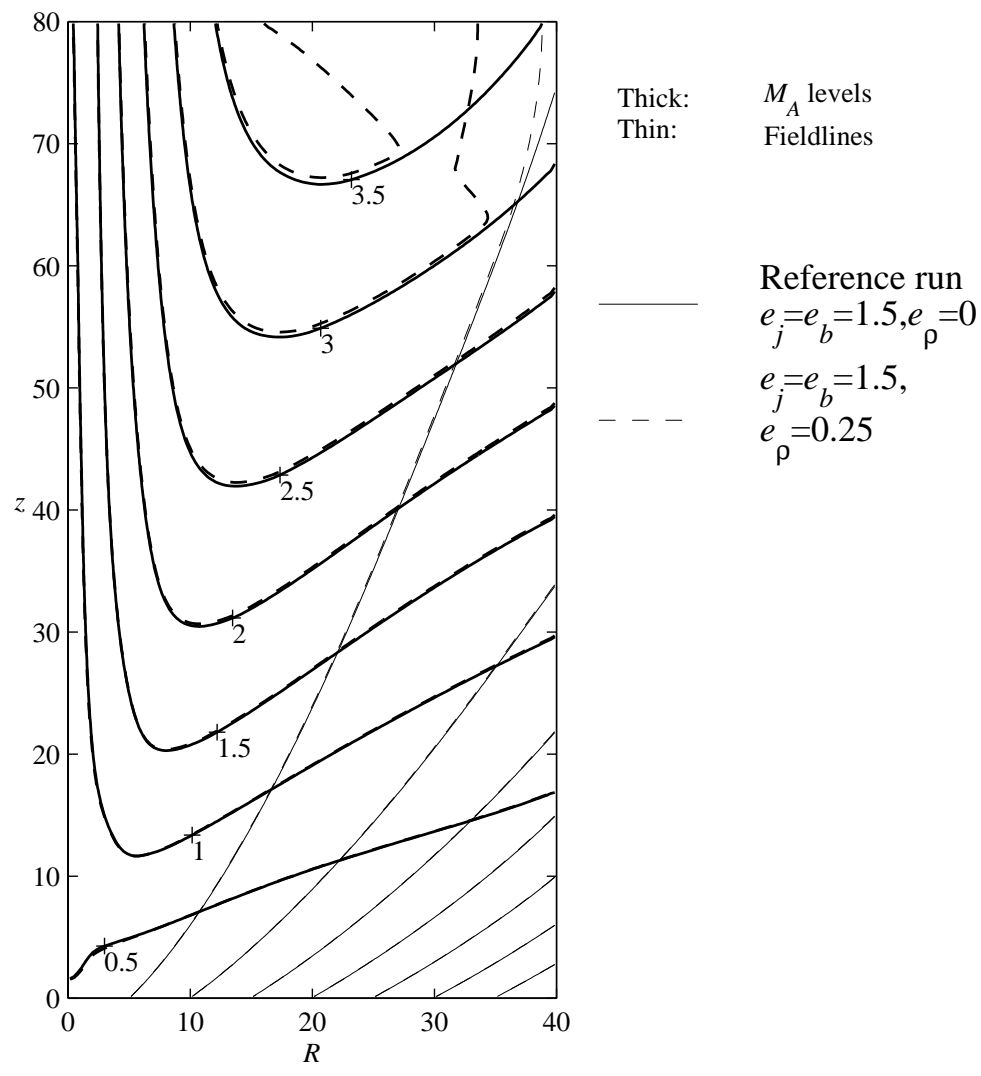

FIG. 6.-Variable density simulation defined by $e_{\rho}=0.25, e_{j}=1.5$, and $e_{\Psi}=-0.5$, is compared to the reference run, which has the same mass flux $j=\rho v_{z}$, and a flat density profile at $z=0$, defined by $e_{\rho}=0.0, e_{j}=1.5$, and $e_{\Psi}=-0.5$. Field lines and contours of $M_{\mathrm{A}}$ in these two runs are shown.

was achieved, we checked that the lower quadrant of this solution was compatible with the reference run. For instance, in Figure 9 we compare the surfaces of constant Alfvén number for the two runs with good results: we recover the strongly accelerated flow found before. The maximum Alfvén number in the lower quadrant of this run is 3.78 , and the maximum fast number is 1.34 , which is close to the reference run and even closer to the results of $\S$ 4.3.1. We also want to check the ratio of projected forces $f_{M} / f_{C}$, especially near the location of the outer corner of the reference run, now the center of the computational box. Looking into Figure 10, we see that $f_{M} / f_{C}$ in this region is smooth, with positive magnetic acceleration; therefore we conclude that the deceleration observed in the reference run was only an edge effect related to the outer corner. As another indication that our treatment of the outer boundary conditions could be improved for $f_{M}$, this simulation shows a tiny triangle of magnetic deceleration near its outer corner.

\subsubsection{Box Size: Change in Width Only; A Sub-Alfvénic Case}

This run has also the same physical parameters, changing only the shape of the computational box. Here we cut the

TABLE 2

DEPENDENCE OF Some Flow QuANTITIES ON THE EXPONENTS $e_{b}, e_{v}$, AND $e_{\rho}$

\begin{tabular}{cccccc}
\hline \hline$e_{v}$ & 1.5 & 0.5 & 1.25 & 0.5 & 1.25 \\
\hline$e_{b} \ldots \ldots \ldots \ldots \ldots \ldots$ & 1.5 & 1.5 & 1.625 & 1.25 & 1.5 \\
$e_{\rho} \ldots \ldots \ldots \ldots \ldots \ldots$ & 0.0 & 0.0 & 0.25 & 0.0 & 0.25 \\
$e_{j} \ldots \ldots \ldots \ldots \ldots \ldots$ & 1.5 & 0.5 & 1.5 & 0.5 & 1.5 \\
$e_{\Psi} \ldots \ldots \ldots \ldots \ldots \ldots$ & -0.5 & -0.5 & -0.375 & -0.75 & -0.5 \\
$\Psi \ldots \ldots \ldots \ldots \ldots \ldots$ & 35.8 & 42.5 & 27.1 & 70.8 & 38.8 \\
$R_{0} \ldots \ldots \ldots \ldots \ldots \ldots$ & 4.77 & 5.64 & 3.91 & 7.68 & 5.15 \\
$R_{A} / R_{0} \ldots \ldots \ldots \ldots$ & 3.29 & 2.31 & 3.03 & 2.61 & 3.31 \\
Max. $M_{\mathrm{A}} \ldots \ldots \ldots$ & 3.98 & 5.06 & 5.26 & 2.66 & 3.78 \\
Max. $v_{p} / v_{K 0} \ldots \ldots$ & 1.67 & 1.33 & 1.67 & 1.31 & 1.54 \\
$\dot{M} \ldots \ldots \ldots \ldots \ldots \ldots$ & 7.73 & 15.02 & 6.89 & 19.54 & 8.05 \\
$L \ldots \ldots \ldots \ldots \ldots \ldots$ & 18.1 & 23.5 & 14.5 & 34.3 & 18.8 \\
$G \ldots \ldots \ldots \ldots \ldots \ldots$ & 54 & 85 & 38 & 162 & 58 \\
$T \ldots \ldots \ldots \ldots \ldots \ldots$ & 5.5 & 8.9 & 4.8 & 10.4 & 5.2 \\
\hline
\end{tabular}

NotEs.-Fluxes and thrust are calculated at the outer corner of the grid, and adimensionalized as in Table 1 . The maxima of $v_{p} / v_{K 0}$ are taken at $z=z_{\max }$. 


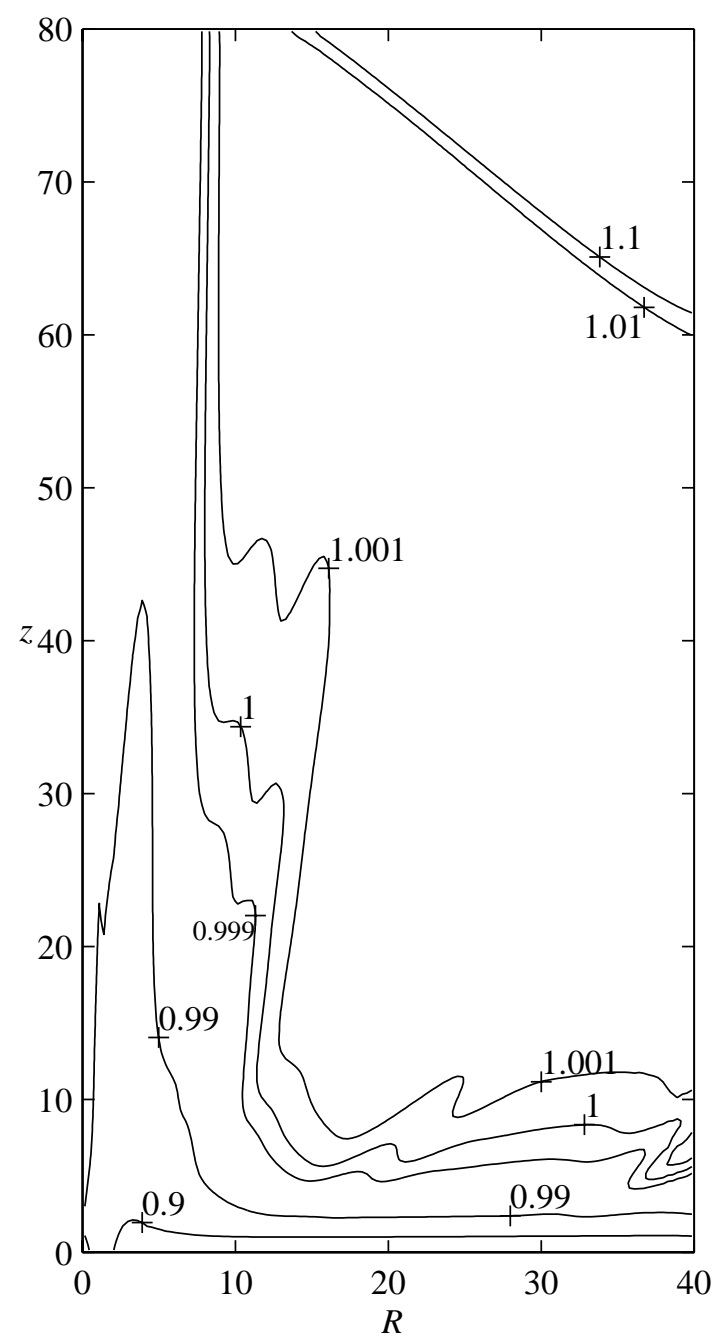

FIG. 7.-Variable density simulation defined in Fig. 6 is again compared to the reference run, this time by showing contours of the density of one run divided by that of the other.

larger box from $\S 4.3 .2$ in half, obtaining a square box with $128 \times 128$ active pixels, $0 \leq z \leq 80.0$, and $0 \leq R \leq 80.0$.

The results show that the Alfvénic surface crosses the outer boundary $z=z_{\max }$ : the flow at the boundary $R=$ $R_{\max }$ is fully sub-Alfvénic. The last field line able to make the Alfvén transition, $\Psi=\Psi_{1}$ crosses now the $z=z_{\max }$ edge. It marks the separation between two very different kinds of simulated flow. Toward the axis, we have the usual magnetocentrifugally accelerated flow in steady state. But outside, in the sub-Alfvénic region, we have a complex structure out of steady state, with filaments of magnetocentrifugal acceleration approximately parallel to the field lines, wide islands where the projected magnetic force $f_{M}$ is negative, and sharp variations in the magnetic twist $B_{\phi} / B_{p}$. Collimation is excessive, slowing down the acceleration of the flow. The total projected force along field lines, $f_{g}+f_{M}+f_{C}$, is negative in a sizable part of the box, instead of only around the axis.

As we have shown in $\S 4.3 .2$, this complex flow disappears in a larger and axially elongated box; it is made possible by the finite size, which sets artificial limits on the critical surfaces. The field lines with $\Psi<\Psi_{1}$ are able to reach the Alfvén surface, carry the proper critical point information, and propagate it downward to the disk. Due to the shape of the box here, most field lines have $\Psi>\Psi_{1}$, with critical points at $z>z_{\max }$, inaccessible to the simulation. Mathematically, the assumptions used to decide the number of functions to fix at the disk surface are wrong, because the critical points are not reached. In this region, each streamline is almost independent from each other, creating the possibility of filaments.

We want to check our assumption that the different behavior of our square and nonsquare boxes is due to the location of the Alfvén surface. To do that, we moved the Alfvén surface used in $\S 4.3 .3$ by reducing the intensity of the magnetic fields by half without changing the box. It allows the flow to become super-Alfvénic inside the square computational box. The flow is smooth, showing again a case where the difference between filamentary and smooth runs lays in the relative position of the simulated Alfvén surface and the computational volume. We have also checked that the conserved quantities are functions of $\Psi$.

\subsubsection{Large Initial $B_{\phi}$ : Dependence on Initial Conditions in the Sub-Alfvénic Case}

The setup used here is similar to the reference run. It differs only in the initial values of $B_{\phi}$ and $v_{\phi}$, set by $v_{\phi}(z=0$, $R)=\left(1-f_{B \phi}\right) v_{K}$ and $B_{\phi}(z=0, R)=f_{B \phi} v_{K} B_{z} / v_{z}$, with $f_{B \phi}$ a small fraction, equal to 0.01 . For simplicity, we have kept using initial values of $B_{\phi}$ and $v_{\phi}$ independent of $z$; we could have chosen them to make the initial $\Omega$ a function of $\Psi$, but the initial values for $B_{\phi}$ would still be away from steady state.

Here we have a large initial toroidal field, which provides excessive collimation of the field lines, making the angle $\theta$ too small for magnetocentrifugal acceleration. Also, the magnetic force along the field lines is such that $\mathscr{B}=\left(R B_{\phi}\right)^{2}$ acts like a pressure; $\mathscr{B}$ grows along the field lines, stopping the acceleration of the flow. The results present filamentation, low acceleration, and high twist. This flow is subAlfvénic in all the volume. We observe here that initial conditions can matter in these simulations, provided that the Alfvén surface is not reached.

\subsubsection{Results of the Convergence Tests}

These tests tell that smooth acceleration of the simulated flow is obtained when a majority of the field lines reach the critical Alfvén surface inside the computational volume. If this condition is met, the simulation results depend mostly on the disk boundary conditions, with little dependence on the outer boundary conditions. The transients are quickly swept up by the advance of the fast accelerating flow. The opposite happens when too many field lines lay in the $\Psi>$ $\Psi_{1}$ region: then the runs show filamentation, excessive collimation, dependence on initial and outer boundary conditions, and - obviously - a slow acceleration. Controlling the position of the critical surfaces inside the computational box can improve convergence, which will help in the setup of future simulations. We plan to utilize this idea in a future work, in which we will set up initial and boundary conditions such that the rotating disk will be enclosed by an Alfvén surface, fully nested inside the computational box; this setup should minimize the influence of the outer boundaries. As shown in $\S 4.3 .3$, the shape and size of the simulation box can sometimes introduce artificial nonsteadiness to a sub-Alfvénic flow. Caution must be observed also in steady state sub-Alfvénic flows, due to their possible dependence on the outer boundary. 


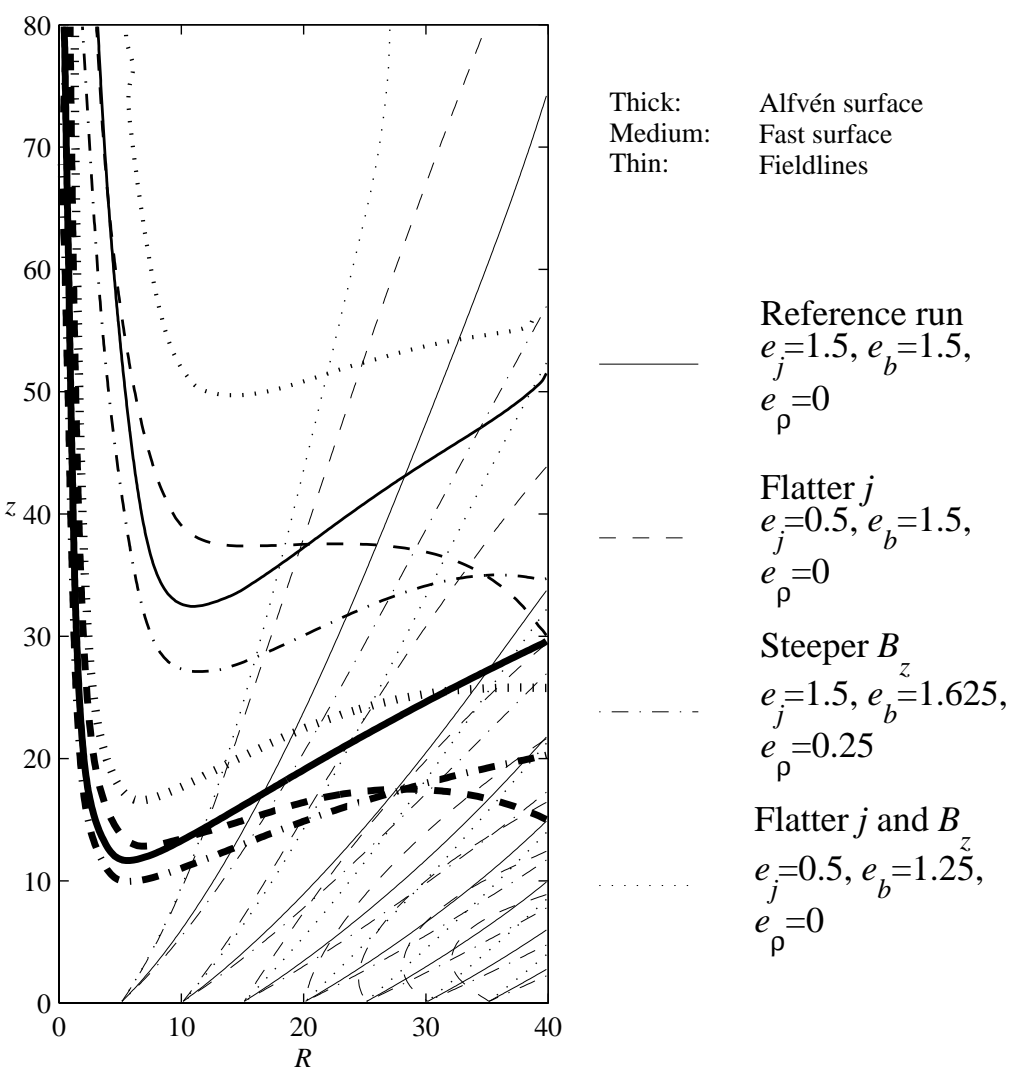

FIG. 8. - Comparison of four runs. Alfvén surface in thick lines, fast surface in medium and field lines in thin. Field lines have been chosen to pass through the same footpoints in the four runs.

The tendencies to filamentation, both of numerical and physical origin, show that the stability and interaction between the various streamlines in this flow are not fully explored yet. This encourages us even more to follow up this work with a three-dimensional simulation, for which this work will be the starting condition. The parallel code necessary to do such massive computation is already written and tested.

\section{CONCLUSIONS}

This paper is the first in a series exploring various aspects of jets launched from accretion disks. Here we have presented the code and shown the results of cold, axisymmetric, time-dependent simulations, paying special attention to the treatment of the jet-launching surface and the region near the rotation axis. From these simulations we conclude that:

1. As expected, cold, steady jets can be launched smoothly from Keplerian disks by the magnetocentrifugal mechanism. The same mechanism is also effective in collimating the outflows. Collimation is observed both in the shape of the field lines and in the density profiles, which become cylindrical and thus jetlike at large distances along the rotation axis, in agreement with asymptotic analysis.

2. The magnetocentrifugal mechanism for jet production is robust to changes in the conditions at the base of the jets, whose consequences are consistent with expectations.
3. Steady state jets obtained in earlier studies using an essentially incomplete treatment of the disk boundary conditions are qualitatively unchanged when this deficiency is rectified. It remains to be shown, however, whether the same is true for nonsteady jets.

4. The structure of the outflows is insensitive to the initial density or flow speed at the injection surface individually, as long as their product (i.e., the mass flux) is kept constant.

5. The size of the simulation box can strongly influence the outcome of a simulation unless most field lines pass through the Alfvén surface within the box. As a result, simulations that produce mainly sub-Alfvénic disk outflows, steady or not, must be treated with caution. This highlights the pressing need for box-invariant simulations, which are under way.

6. Zeus3D can be parallelized so that solutions from different subgrids match smoothly.

This research was performed in part using the CACR parallel computer system operated by Caltech. We acknowledge useful discussions with James Stone, John Hawley, David Meier, and Shinji Koide. This research was supported by NSF grant AST 95-29170 and NASA grant 5-2837. R. B. thanks John Bahcall for hospitality at the Institute for Advanced Study and the Beverly and Raymond Sackler Foundation for support at the Institute of Astronomy. 


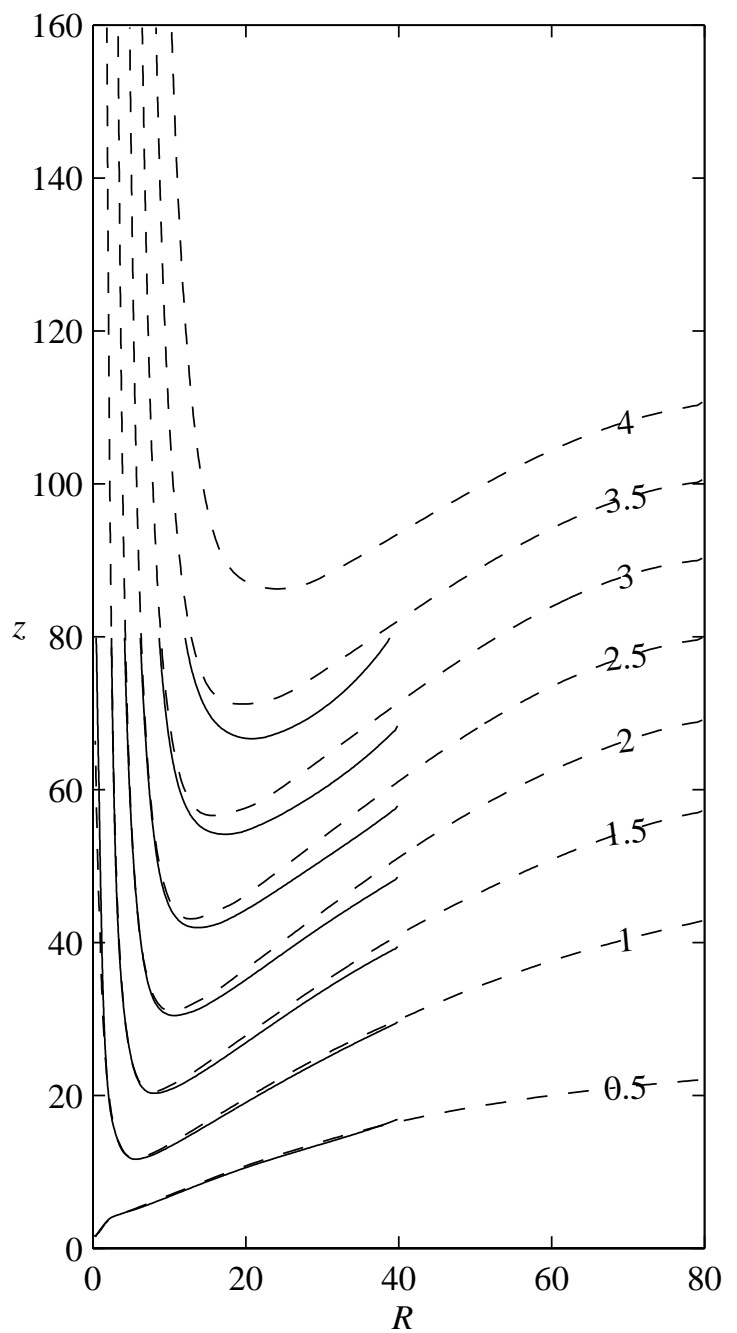

FIG. 9.- Contour plots of $M_{\mathrm{A}}=v_{p} / v_{A p}$, for the reference run (solid lines) and a larger and wider box (dashe lines), compared to check convergence.

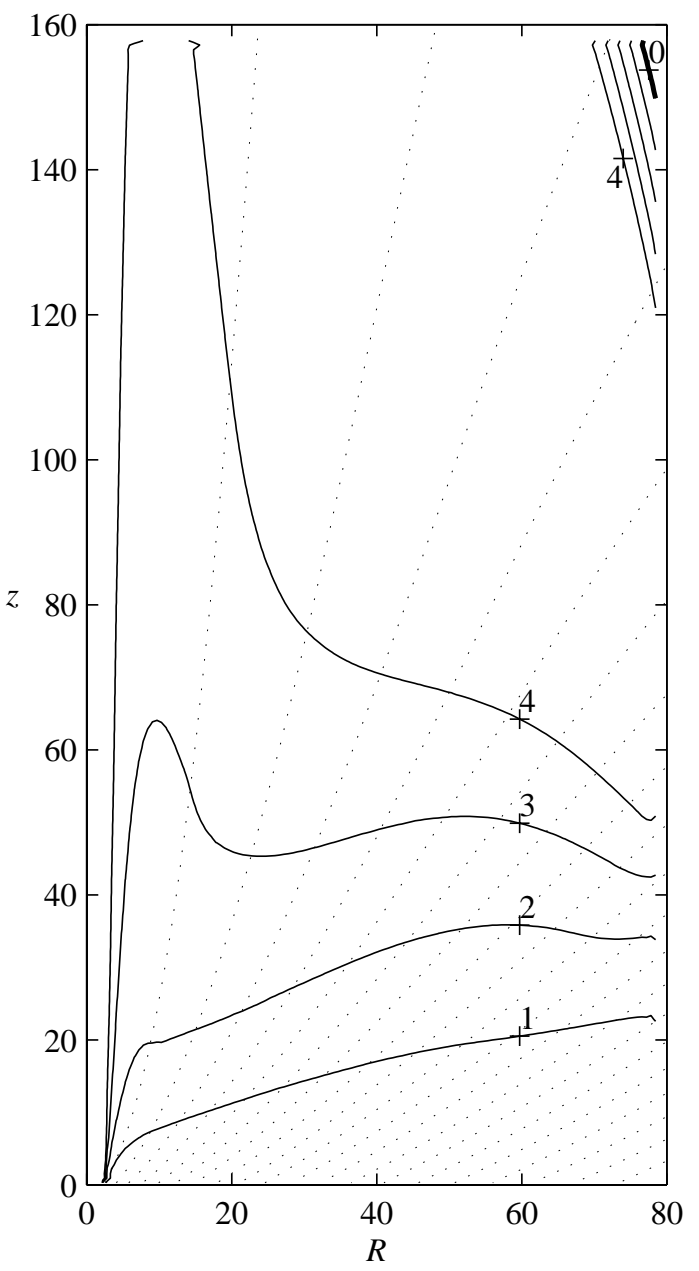

Fig. 10.- Simulation on the larger and wider box. In solid, the contour levels $f_{M} / f_{C}=0,1,2,3$, and 4 , show the ratio of magnetic over centrifugal forces along the field lines (dotted lines). The $f_{M}=0$ level is a short, thick line at the outer corner.

\section{APPENDIX}

\section{TWO NUMERICAL ARTIFACTS RELATED TO BACKFLOW}

We mention here some numerical problems that had to be overcome in developing the code, in the spirit of helping developers of similar codes. A simulation can develop backflow through at least two physical mechanisms. One is simple gravitational infall, which operates mostly in the axial region, where the magnetocentrifugal acceleration fails. A second mechanism is cocoon backflow. Due to the differential Keplerian rotation, the inner parts of the flow launch a jet earlier than the outer. The cocoon of this jet may produce a temporary backflow. When the backflow impinges into the disk boundary, our boundary conditions shown in $\S 3.2 .4$ carefully allow the disk to absorb it. This easy prescription avoids the problems discussed below. However, in our earliest simulations, our disk boundary was independent of the sign of $v_{z}$ in the first active zone. This created two kinds of trouble: false acceleration and filamentation.

\section{A1. FALSE ACCELERATION}

In this scenario, backflowing material tries to get back toward the disk, where it finds a boundary condition imposing a positive speed outward. Compression increases the density close to $z=0$. This increased mass is then flung out at the speed $v_{z}$ given by the boundary condition imposed. No physical process is involved: only a badly chosen boundary. This false acceleration is seen more clearly when we reduce the axial injection, allowing the gravitational infall to go all the way into the disk; with incorrect boundary conditions, it quickly bounces back unphysically.

\section{A2. NONPHYSICAL FILAMENTATION}

Let's suppose now that, early in the simulation, a portion of the cocoon backflow touches the disk at some radius $R=R_{1}$. If the disk boundary condition insists on imposing a forward speed $v_{z}>0$ to the material, we will get an increase in density due to the artificial compression of the coronal material. If the ejection speed $v_{z}$ is not large, a fast bounce-back will be avoided, 
and we will not get a false acceleration like before. Nevertheless, we still get a localized region, close to the disk, where the density is abnormally large. This larger density will be associated with large localized $B_{\phi}$, and it will have a large inertia; the magnetocentrifugal acceleration will not be enough to lift the material up. The region around $R_{1}$ will be a hole in the acceleration front; the jet will proceed forward for both smaller and larger radii, but it will stagnate here. A succession of such holes would make the simulation look filamentary; again, allowing the disk to absorb the backflow avoids this numerical effect.

\section{REFERENCES}

Begelman, M. C., Blandford, R. D., \& Rees, M. J. 1984, Rev. Mod. Phys., 56,255

Blandford, R. D., \& Payne, D. G. 1982, MNRAS, 199, 883

Blandford, R. D., \& Znajek, R. L. 1977, MNRAS, 179, 433

Bogovalov, S. V. 1997, A\&A, 323, 634

Bogovalov, S. V., \& Tsinganos, K. 1999, MNRAS, 305, 211

Clarke, D. A. 1992, EDITOR User Manual, ftp://zeus.ncsa.uiuc.edu/LCA/ codes/zeus3d/editor/manuals/editor21.tex

Clarke, D. A., Norman, M. L., \& Burns, J. O. 1986, ApJ, 311, L63

Clarke, D. A., Norman, M. L., \& Fiedler, R. A. 1994, Zeus3D User Manual, $\quad \mathrm{ftp}: / /$ zeus.ncsa.uiuc.edu/LCA/codes/zeus3d/manuals/ zeus32.tex

Evans, C. R., \& Hawley, J. F. 1988, ApJ, 332, 659

Fiege, J. D., \& Henriksen, R. N. 1996, MNRAS, 281, 1038

Hawley, J. F., \& Stone, J. M. 1995, J. Comput. Phys., 89, 127

Heinemann M., \& Olbert, S. 1978, J. Geophys. Res., 83, 2457

Henriksen, R. N., \& Rayburn, D. R. 1971, MNRAS, 152, 323

Hjellming, H. M. 1997, in ASP Conf. Proc. 121, Accretion Phenomena and Related Outflows, ed. D. T. Wickramasinghe, G. V. Bicknell, \& L. Ferrario (San Francisco: ASP), 53

Keppens, R., \& Goedbloed, J. P. 1999, A\&A, 343, 251

Königl, A. 1989, ApJ, 342, 208

Königl, A., \& Pudritz, R. E. 2000, in Protostars and Planets IV, ed. V. Mannings, A. Boss, \& S. Russell (Arizona: Univ. Arizona Press), in press (astro-ph/9903168)

Lada, C. J. 1985, ARA\&A, 23, 267

Lind, K. R., Payne, D. G., Meier, D. L., \& Blandford, R. D. 1989, ApJ, 295, 358

Livio, M. 1997, in ASP Conf. Proc. 121, Accretion Phenomena and Related Outflows, ed. D. T. Wickramasinghe, G. V. Bicknell, \& L. Ferrario (San Francisco: ASP), 845

Lovelace, R. V. E., Mehanian, C., Mobarry, C. M., \& Sulkanen, M. E. 1986, ApJS, 62, 1

Meier, D. L., Edgington, S., Godon, P., Payne, D. G., \& Lind, K. R. 1997, in ASP Conf. Proc. 123, 12th Kingston Meeting: Computational Astrophysics, ed. D. A. Clarke \& M. J. West (San Francisco: ASP), 290

Mestel, L. 1968, MNRAS, 138, 359

Mirabel, I. F., \& Rodríguez, L. F. 1998, Nature, 392, 673

Najita, J. R., \& Shu, F. H. 1994, ApJ, 429, 808

Norman, M. L. 1996, The ZEUS3D Code, http://zeus.ncsa.uiuc.edu:8080/ lca intro zeus3d.html

Ostriker, E. C. 1997, ApJ, 486, 291

Ouyed, R., \& Pudritz, R. E. 1997, ApJ, 482, 712

Pudritz, R. E., \& Norman, C. A. 1986, ApJ, 301, 571

Romanova, M. M., Ustyugova, G. V., Koldoba, A. V., Chechetkin, V. M., \& Lovelace, R. V. E. 1997, ApJ, 482, 708

Sakurai, T. 1985, A\&A, 152, 121 1987, PASJ, 39, 821

Shu, F. H., Najita, J., Ostriker, E. C., \& Shang, H. 1995, ApJ, 455, L155

Shu, F. H., Ruden, S. P., Lada, C. J., \& Lizano, S. 1991, ApJ, 370, L31

Stone, J. M., \& Norman, M. L. 1992a, ApJS, 80, 753 1992b, ApJS, 80, 791 1993a, ApJ, 413, 198 1993b, ApJ, 413, 210

Ustyugova, G. V., Koldoba, A. V., Romanova, M. M., Chechetkin, V. M., \& Lovelace, R. V. E. 1999, ApJ, 516, 221 